\title{
Perspectivas da municipalização do controle da filariose linfática na região metropolitana do Recife
}

\author{
Views on the municipalization of lymphatic filariasis \\ control in greater metropolitan Recife
}

Amélia Maciel 1

AndréF. Furtado 1

Keyla B. F. Marzochi 2

\footnotetext{
1 Centro de Pesquisas Aggeu Magalhães/Fiocruz, Universidade Federal de Pernambuco. C. P. 7472, Recife, PE 50670-420, Brasil.

2 Escola Nacional de Saúde Pública/Fiocruz. Av. Leopoldo Bulhões 1480 , Rio de Janeiro, RJ 21040-210, Brasil.
}

Abstract Lymphatic filariasis is caused by the nematodes Brugia malayi, Brugia timori, and Wuchereria bancrofti. The disease occurs in devel oping countries and is more frequent in urban areas. An estimated 4 billion people live in at-risk areas. In Brazil the endemic is caused by W. bancrofti and was first documented in 1878. It was first detected in Recife in 1952. Currently, Recife and Belém are the only cities in Brazil wherethe endemic is considered a public health problem. The objectives of this study are to discuss the epidemiology and control of Iymphatic filariasis and review its control since it was reported by Rachou in 1952. We analyze the "campanhista" or campaign-oriented model employed by the Sucam/FNS institutional program, as well as several proposed innovative methods. We present available strategies for control of filariasis through primary health care services, decentralization to thelocal level (or "municipalization"), and community-based health programs.

Key words Lymphatic Filariasis; Filariasis; Wuchereria bancrofti; Communicable Disease Control

Resumo A filariose linfática resulta da infecção por três nematódeos: Brugia malayi, Brugia timori e Wuchereria bancrofti. Atingindo países considerados em desenvolvimento, é mais distribuída em áreas urbanas. Estima-se que quatro bil hões de pessoas vivem em áreas de risco. No Brasil, a endemia é causada por W. bancrofti efoi documentada em 1878; na cidade do Recife, foi detectada em 1952. Atualmente, apenas na regi ão metropolitana do Recife e Belém, esta endemia é considerada um problema de saúde pública. Neste trabal ho objetivamos abordar e discutir a questão da epi demi ol ogia e controle da filariose linfática erevisar o histórico de seu control e desde sua notifi cação em 1952 por Rachou, até nossos dias. Analisamos o model o campanhista do programa institucional da Sucam/FNS e os métodos al ternati vos atuais propostos como inovadores. Apresentamos estratégias viáveis para o controle da filari ose, através da rede primária de saúde, da muni ci palização e do Programa de Agentes Comunitári os de Saúde (Pacs). Palavras-chave Filariose Linfática; Filariose; Wuchereria bancrofti; Controle de Doenças Transmissíveis 


\section{Introdução}

A filariose linfática no Brasil foi registrada pela primeira vez em 1877 (Azevedo, 1955). Desde o primeiro inquérito realizado no País em 1952, Recife e Belém foram considerados os principais focos da endemia (Rachou, 1957), a qual, ainda hoje, permanece como um problema de saúde pública. Após esse inquérito, foi estabelecido o Programa Nacional de Controle da Filariose, sob a responsabilidade do então Departamento Nacional de Endemias Rurais (DNERu), que depois passou a se chamar Superintendência de Campanhas (Sucam), e, atualmente, Fundação Nacional da Saúde (FNS).

Segundo dados mais recentes do boletim da Sucam, as áreas de filariose bancroftiana de interesse para a saúde pública no Brasil encontram-se ainda nos estados do Pará e Pernambuco, com índices microfilarêmicos de 0,02\% e $4,9 \%$, respectivamente. O mesmo boletim relata também que focos endêmicos foram encontrados em Alagoas, onde, de 10.959 pessoas examinadas, 104 (0,95\%) eram microfilarêmicas (MS, 1992).

Na cidade do Recife, Wuchereria bancrofti foi documentado em 1952 (Azevedo \& Dobbin J r., 1952), no bairro de Afogados e, desde então, durante mais de quarenta anos, vêm sendo realizados levantamentos epidemiológicos, seguidos de tratamento individual dos microfilarêmicos. De 1980 a 1990, cinqüenta mil pessoas foram diagnosticadas e tratadas com dietilcarbamazina (DEC), seja nas campanhas casa a casa (busca ativa), seja nos postos de saúde (postos de notificação passiva), tendo sido distribuídos 3.686.154 comprimidos (MS, 1990).

Em 1982, em um estudo analítico dos dados secundários da Sucam, distribuindo-se a prevalência da filariose por estrato sócio-econômico, constatou-se que a prevalência, em certas áreas ou bairros do Recife, estava aumentando (Morais, 1982). No ano seguinte, a Sucam referia que a endemia atingira índices satisfatórios, favoráveis ao seu controle (MS, 1983).

Em 1985, foi estabelecido no Centro de Pesquisas Aggeu Magalhães/ Fiocruz (CPqAM) o Programa de Filariose em Recife, em decorrência da demanda intensa a esse serviço de pacientes com formas clínicas da doença.

Entre 1989 e 1991, foi realizado um inquérito epidemiológico em várias áreas do Recife, tendo sido analisadas quinze mil pessoas e encontradas prevalências variando de 1,5 a $15 \%$, com uma média de 6,5\% (Maciel et al., 1996). Naquele mesmo período, inquéritos epidemiológicos na cidade de Olinda (região metropoli- tana do Recife) revelaram índices microfilarêmicos maiores que os de Recife, com uma prevalência de 12,3\% (Maciel et al., 1994).

Altas taxas de microfilaremia e densidade parasitária associadas a baixos índices de formas agudas e crônicas refletem aumento de transmissão ou transmissão recente (Grove, 1983).

Medeiros et al. (1992) referem que o programa institucional de controle da filariose da FNS em Recife havia diminuído os índices de infectividade do vetor Culex quinquefasciatus, quando comparados com aqueles observados em Olinda, em conseqüência das campanhas e tratamentos sistemáticos de indivíduos microfilarêmicos realizados no passado.

O padrão epidemiológico por nós encontrado aponta maior densidade média em Olinda, $70 \mathrm{mf} / 60 \mu \mathrm{l}$ de sangue por indivíduo, contra $65 \mathrm{mf} / 60 \mu \mathrm{l}$ de sangue por indivíduo em Recife. Os valores encontrados, quase idênticos, reforçam os achados de Jayasekera et al. (1991), demonstrando que tais índices, tanto em Recife, quanto em Olinda, são ideais para transmissão.

\section{O controle da filariose linfática no Recife}

\section{Histórico}

Nos anos 40, foram criados vários serviços nacionais de controle de endemias, como o Serviço Nacional de Febre Amarela (SNFA) e o Serviço Nacional de Malária (SNM). Em 1956, surgiu o DNERu, que agrupou todos os demais serviços.

O controle da filariose linfática no Brasil iniciou-se na década de 50, cabendo a René Rachou a coordenação da campanha. Inicialmente foi realizado um levantamento epidemiológico para avaliar a distribuição geográfica da endemia em todo o país e identificar os vetores da doença (Rachou, 1954).

Em um período de dez anos, após a conclusão de vários inquéritos e de terem sido implementadas ações visando a eliminação de focos endêmicos em Manaus, Maceió, Salvador, Florianópolis e Porto Alegre, constatou-se que apenas Recife e Belém persistiram como focos endêmicos importantes que requeriam programas de controle permanentes. Em 1957 foi estimado que no Recife havia 80.000 microfilarêmicos e 50.000 em Belém (Rachou, 1957).

O programa nacional de controle do vetor da filariose foi iniciado em 1952, com inseticida de efeito residual, o hexaclorobenzeno (BHC), ao mesmo tempo em que se fazia o tratamento 
dos portadores de microfilaremia (Scaff \& Gueiros, 1967).

Em 1953, com a demonstração da ineficácia do BHC, uma nova estratégia de controle do vetor foi idealizada, baseada no controle das formas aquáticas (larvas). Como este método de controle tornou-se dispendioso, pois requeria aplicações semanais, foi abandonado.

Em 1955, surge um novo inseticida residual, o dieldrin, que também se mostrou ineficaz contra o C. quinquefasciatus. Diante da inexistência de inseticida eficaz contra o mosquito adulto e por causa dos altos custos dos métodos larvicidas, abandonou-se a estratégia de combate ao vetor, iniciando-se o ciclo de atenção ao homem, tendo como base o tratamento dos microfilarêmicos (Scaff \& Gueiros, 1967).

A droga terapêutica utilizada foi a 1-dietilcarbamil 4 metilpiperazina (DEC), empregada no Brasil com o nome comercial de Hetrazan. Azevedo (1955) usou a medicação nos indivíduos microfilarêmicos, referindo tratar-se de "arma profilática para redução da mi crofilare mia" (Azevedo, 1955). Em 1958, Rachou \& Scaff (1958) reportam os primeiros resultados da ação do Hetrazan em Belém, utilizando diferentes esquemas da mesma dose de $6 \mathrm{mg} / \mathrm{kg} /$ dia por 21, 15, dez e sete dias consecutivos de tratamento. Os mesmos autores referem ainda que se poderiam utilizar esquemas de curta duração para campanhas de tratamento em massa, administrando-se a mesma dosagem em um só dia, com periodicidade semestral ou anual, para um efetivo controle da endemia.

Após observações de longa duração, verificando-se que o esquema de tratamento em dose única não atingira os efeitos esperados, optou-se pelo esquema de tratamento com $6 \mathrm{mg} /$ $\mathrm{kg} /$ dia por sete dias consecutivos (Franco \& Lima, 1967).

Nos anos 70, reformas no Ministério da Saúde fizeram com que o DNERu, o SNFA e o SNM fossem agregados em uma única entidade, a Sucam, que passaria a ser responsável pelo combate a endemias transmitidas por vetores. Esta procuraria, a curto prazo, incorporar as medidas tradicionais exitosas, tais como os inquéritos de busca ativa associados ao tratamento dos pacientes microfilarêmicos, e, a longo prazo, instalaria postos de notificação passiva em áreas estratégicas para atenção primária a pacientes microfilarêmicos e com formas agudas e crônicas.

\section{Estratégias e métodos}

Atualmente, o programa de controle da filariose bancroftiana proposto pelo CPqAM está baseado no tratamento em massa da população das áreas de risco, com doses baixas e espaçadas da DEC, na redução do contato do vetor com o homem (uso de mosquiteiros, telagem das janelas) e na redução da densidade de vetores nas áreas endêmicas (eliminação de criadouros reais e potenciais, vedação de fossas sépticas). Tais medidas são fundamentais, já que o saneamento básico é ausente nas áreas de baixa renda, ou, quando existente, é ineficiente em relação à drenagem de dejetos.

No período de 1982 a 1992, dos investimentos realizados no programa de saneamento básico, envolvendo fornecimento de água, esgotamento sanitário e coleta de lixo, a maior parcela de recursos coube à região Sudeste, com $44,04 \%$ do orçamento previsto; apenas 4,5\% destinou-se à região Nordeste. Para a implementação de programas de saneamento básico em áreas urbanas de todo o país, seriam necessários US\$70.60 por habitante, para esgotamento sanitário, e US\$38.50 por habitante, para melhorias sanitárias (Cabes, 1994). A região metropolitana do Recife tem uma população estimada em três milhões de habitantes, e um programa dessa monta, apesar de oneroso, contribuiria para a redução de outras doenças associadas à fal ta de infra-estrutura sanitária, como diarréias, dengue, leptospirose, cólera e hepatite, contribuindo, também, para a redução drástica da mortalidade infantil.

Durante quase cinqüenta anos, a DEC foi o único recurso terapêutico utilizado para tratamento da filariose linfática. É uma droga segura e eficiente, sendo absorvida pelo trato gastrointestinal, com excreção renal; não tem ação teratogênica ou abortígena, e seu mecanismo de ação é mediado por anticorpos e plaquetas.

Em virtude da ação parasiticida da DEC, a morte das mf durante o tratamento pode ocasionar efeitos colaterais capazes de provocar reações sistêmicas e locais, como febre, vômitos, cefaléia e hematúria.

Entre as formas de distribuição da droga para a população, pode ser indicado o tratamento seletivo, utilizando-se a dose padrão de $6 \mathrm{mg} / \mathrm{kg} / \mathrm{dia}$, durante 12 dias; o tratamento em massa com baixas doses (WHO, 1988); ou ainda o uso do sal de cozinha medicado com DEC (Ottesen \& Ramachadran, 1995). Um outro recurso terapêutico contra a filariose disponível atualmente é a ivermectina, um antibiótico semi-sintético derivado da ivermectina B1 macro-cíclico-lactone, produzido pelo actinomi- 
ceto Streptomyces avermitilis. Empregado largamente na prática veterinária para tratamento de parasitos, vem sendo usado para o tratamento da oncocercose com relativo sucesso desde 1982.

Para o controle da filariose bancroftiana, a ivermectina já foi utilizada em estudos-piloto no Sri Lanka, Haiti, Índia, Polinésia e Senegal. No Brasil, foi realizado um estudo-piloto em militares da região metropolitana do Recife (Coutinho et al., 1994). Os autores concluíram que a ivermectina é um potente microfilaricida, porém, na avaliação do programa, verificaram que os pacientes microfilarêmicos recidivaram, apresentando índices superiores a 20\% após seis meses. Isto demonstra que a droga tinha apenas efeito microfilaricida, ao contrário da DEC, que tem ação macrofilaricida (Ottesen, 1985; Furtado et al., 1994). Além disso, as manifestações adversas da ivermectina são, de longe, mais intensas e freqüentes do que as ocasionadas pela DEC (Coutinho et al., 1994).

No que concerne à redução ou eliminação do vetor, as medidas disponíveis incluem ações contra o inseto adulto e formas jovens (larvas), usando-se métodos físicos, químicos e biológicos.

Programas de controle utilizando inseticidas organoclorados, organofosforados piretroides ou outros, em aplicações espaciais ou de efeito residual, têm sido abandonados nos anos recentes, em razão, principalmente, dos gastos exorbitantes gerados pela necessidade de aplicações freqüentes de acordo com o ciclo biológico dos insetos. Outros fatores que também levaram ao abandono dos programas de controle são a redução dos efeitos esperados por causa do uso indiscriminado daqueles inseticidas, o que causou resistência, bem como os efeitos nocivos à fauna não-alvo, ao meio ambiente, ao homem e outros vertebrados (Curtis, 1994).

Bactérias do gênero Bacillus, B. thuringiensis var. israelensis e B. sphaericus produzem toxinas que têm ação potente contra larvas de C. quinquefasciatus.

Estudos-piloto utilizando B. thuringienses var. israelensis, visando ao controle de culicídeos e simulídeos (borrachudo), têm sido desenvolvidos em países como Cuba, Canadá, França, Israel, Nigéria, Suíça e Estados Unidos da América (Barjac \& Sutherland, 1990).

Tem sido demonstrado que cepas de B. sphaericus, especialmente as cepas 1.593 e 2.362 , são mais eficientes do que as de $B$. thuringiensis para o controle de $C$. quinquefasciatus, por apresentarem maior tempo de persistência nos habitats típicos de larvas do vetor
(Mulla et al., 1984; Regis et al., 1994). Podemos citar ainda a utilização de peixes larvívoros, como Gambusia spp., como um importante agente de controle biológico. A eficácia dos agentes biológicos no controle de $C$. quinquefasciatus, depende de fatores ambientais, como a qualidade da água dos criadouros, o clima, a densidade larvária (Regis et al., 1994). Portanto, antes de se empregar qual quer medida de controle do vetor, estudos-piloto devem ser realizados para avaliação e padronização da dose e da freqüência das aplicações, a fim de se obterem respostas eficientes e eficazes.

O método das pérolas de poliestireno (bolinhas de isopor) tem importância bastante destacada, por ser um método barato (US\$ 5,00 por cada fossa séptica), seguro para quem o aplica e para a população-alvo em geral, com a vantagem de ser aplicado manualmente, sem anteparos sofisticados ou necessidade de treinamento especializado. Experiências de campo realizadas na Tanzânia reduziram o número de picadas de mosquitos por ano por pessoa, de 24.993 para 439, após a aplicação do método (Maxwell et al., 1990). No Recife, a redução foi de mil para menos de três mosquitos por fossa, após o uso das pérolas de poliestireno. A freqüência de suas aplicações é pequena, estimando-se sua permanência por um mínimo de cinco anos, sem que haja necessidade de reposição das pérolas (Regis et al.,1995).

Novas abordagens para antigas propostas: perspectivas para a municipalização

Com o objetivo de controlar e solucionar problemas de saúde pública, foram idealizados os programas de controle de endemias, como malária, febre amarela e doença de Chagas, estruturados e organizados em sua origem segundo o modelo campanhista (verticalizado e centralizado). Este modelo, desenvolvido no início deste século, fundamentava-se, em seus aspectos teórico e filosófico, na importância, para a população brasileira, de epidemias como febre amarela e peste, cuja gravidade e extensão era imensa perante as autoridades sanitárias de então, com impacto econômico em relação às atividades comerciais e produção de bens e serviços, comprometendo o interesse do desenvolvimento nacional e a expansão do capital interno e mercantil. No aspecto prático, as ações de saúde concentravam-se nos centros urbanos, eram limitadas no tempo e espaço, tinham um caráter bastante autoritário e disciplinado (MS, 1994). 
Nas décadas de 20 e 30, as campanhas contra a febre amarela e a malária, em cooperação bilateral com a Fundação Rockfeller, obtiveram êxito nos centros urbanos, principalmente no Nordeste brasileiro, resultando na erradicação do Anopheles gambiae, vetor da malária. Entretanto, em relação às doenças crônicas, como tuberculose e ancilostomíase, o resultado não foi o mesmo. Na década de 40 , foram criados serviços nacionais específicos de controle de doenças como hanseníase, tuberculose e peste, os quais se diferenciavam das campanhas anteriores pelos novos modelos organizacionais, sem a disciplina rígida daquelas.

O modelo campanhista teve grande eficácia durante algumas décadas de execução desses programas, porém, com o passar dos anos, não alcançaram mais os objetivos para os quais tinham sido criados. A deficiência de um investimento adequado repercutindo sobre a manutenção e aperfeiçoamento dos recursos humanos, assim como a persistência de práticas e métodos superados, geraram distanciamento dos resultados previstos no programa. Em relação ao nível técnico, faltou o investimento em novas estratégi as, fundamentadas em conhecimento e pesquisa, capazes de superar os problemas detectados, como o da resistência do Plasmodium fal ci parum a medicamentos, ou o da inexistência de inseticidas que contemplassem a especificidade das áreas trabal hadas em seu componente social e ambiental. Além disso, as metodologias em geral precisavam se adequar aos novos tempos, em face das dificuldades logísticas e operacionais de diversos programas e de novas estratégias de controle (MS, 1994).

Para uma melhor otimização das intervenções e ações de controle faz-se necessário uma reavaliação técnica dos programas, em razão da especificidade das doenças, vetores, reprodução e transmissão. Nesse sentido, é de interesse a busca de técnicas e modelos alternativos para uma melhor eficácia. Contudo, continua, ainda hoje, nesses programas, a preponderância de marcos teóricos antigos, concernentes aos programas originais do passado.

A reformulação dessas ações assume maior importância diante da compreensão atual das endemias enquanto processos coletivos e não apenas como agravos individuais, com determinação biossocial, dependente não somente do vetor/parasito, mas também da forma como os grupos sociais (comunidades) se inserem e se reproduzem na sociedade maior, gerando condições ideais para a transmissão e manutenção do ciclo epidemiológico. No caso de certas endemias, em que a filariose pode ser também um modelo, fatores como a concentração populacional, a criação e manutenção dos vetores, a percepção não prioritária da comunidade sobre a doença, associadas ao cenário urbano, com precárias condições de saneamento, habitação, além da migração intensa entre áreas urbanas, assistência e diagnóstico clínico-laboratorial pouco acessíveis e às vezes de baixa sensibilidade, requerem análise e intervenção de caráter interdisciplinar.

Priorizando a questão da municipalização, é necessário reformular e redimensionar as ações de saúde em relação à filariose linfática como processo e expressão coletivos dentro de uma comunidade endêmica, sem limitar-se à identificação de indivíduos microfilarêmicos, seja no método campanhista ou nos postos de notificação passiva já distribuídos na região metropolitana do Recife, seja no que concerne ao diagnóstico das formas crônicas e agudas da doença. Quanto ao diagnóstico, seus objetivos não al cançaram uma importante fatia de portadores da endemia com formas clínicas agudas e crônicas, como linfedema, hidrocele e elefantíase, que foram relegadas a segundo plano, quando não esquecidas totalmente.

Em relação às ações de controle da filariose, o município teria o papel de execução, avaliação e supervisão, porém o que constatamos atualmente é que estamos vivenciando ações e programas do passado, incluindo o uso de inseticidas organofosforados como o Fenthyon (Lebacyd $\left.{ }^{\circledR}\right)$, que, além de desenvolver resistência, tem efeito de curta duração sobre populações de Culex quinquefasciatus e cuja toxicidade não é seletiva. É um método superado, que causa prejuízos ao meio ambiente e à população humana. Sua indicação para a aplicação em massa parece fundamentada no discurso de que se constitui em “um método de impacto ealívio rápido das muriçocas para as populações"; todavia, sabe-se que leva a resultados frustrados e danosos, se comparado a outras al ternativas técnicas de controle atuais, inovadoras, inócuas para as espécies não-alvo, não poluentes e extremamente eficientes.

O papel do Estado na questão do controle da filariose seria o de coordenar informações, efetuar a avaliação epidemiológica e garantir o apoio laboratorial às atividades de controle e vigilância (MS, 1994). O Estado, em sua função gestora, assume a realização de seminários, com a apresentação de uma proposta para controle da filariose, em municípios da região metropolitana do Recife. Dessa forma, afirma o seu papel de articulador intersetorial em nível de outras instituições envolvidas no controle da doença, reforçando a importância da conti- 
nuidade das ações programadas, para obter uma melhor eficácia das atividades de controle (SES, 1996).

O verdadeiro salto de qualidade consistiria não em questionar a quem a filariose pertence ou é designada, mas em reformular os atuais programas, a fim de conceder-Ihes uma dimensão setorial, onde seus objetivos, devidamente redimensionados, sejam perseguidos mediante a conjugação dos recursos financeiros, tecnológicos, materiais e humanos da União, dos estados e dos municípios envolvidos. No contexto do SUS, tratar a questão das endemias significa muito mais do que simplesmente buscar viabilizar a transferência de serviços ou de ações de controle de uma esfera de governo para outra (MS, 1994).

\section{A persistência da endemia e a questão do controle}

A OMS sugere que o controle da filariose linfática seja realizado ou integrado ao sistema de atenção primária à saúde, tanto municipal como estadual. Desde 1989, o programa de controle da FNS está metodologicamente baseado na verificação de indivíduos microfilarêmicos das áreas reconhecidamente endêmicas, mediante coleta da gota espessa; no tratamento dos microfilarêmicos com DEC, posologia de $6 \mathrm{mg} / \mathrm{kg} /$ dia por 12 dias; e na instalação dos postos de notificação passiva em cinco municípios da região metropolitana do Recife (Recife, Olinda, Paulista, Jaboatão e I garassu) (Tabela 1).

A abordagem do controle institucional da FNS baseia-se exclusivamente no tratamento seletivo com DEC dos indivíduos microfilarêmicos. Sabemos que a DEC pode levar alguns microfilarêmicos a desconforto, tanto pela sua

Tabela 1

Índices de microfilaremia na região metropolitana do Recife, ano de 1989.

\begin{tabular}{lrc}
\hline Município & Pessoas examinadas & Microfilaremia \\
\hline Recife & 95.467 & 2,1 \\
Olinda & 118.173 & 1,9 \\
Jaboatão & 4.081 & 2,2 \\
São Lourenço da Mata & 1.985 & 0,3 \\
Paulista & 2.249 & 0,4 \\
Igarassu & 72 & 0,0 \\
Moreno & 54 & 0,0
\end{tabular}

Fonte: MS, 1990. toxicidade, quanto pelas reações provocadas pela morte das microfilárias, induzindo os mesmos à interrupção e até ao abandono do tratamento.

Na nossa experiência clínica, de 356 indivíduos microfilarêmicos tratados e acompanhados durante o período de seis meses, $44,7 \%$ completaram o tratamento sem reações adversas; $38,2 \%$ tiveram reações colaterais e $17,1 \%$ abandonaram o tratamento. Não existem dados confiáveis pertinentes ao abandono do tratamento empregado pelo Programa de Controle da FNS. Os microfilarêmicos que persistem positivos mantêm a cadeia epidemiológica da transmissão, assegurando, assim, os níveis anteriores de prevalência.

Outra fal ha do programa realizado pela FNS, está relacionada ao diagnóstico da parasitose, tanto pela quantidade de sangue coletado, como pelo horário de coleta, já que a gota espessa é o único recurso disponível para o diagnóstico em nível municipal e federal. Esta fal ha foi por nós constatada comparando dois períodos de coleta com práticas diferentes. Durante o período de 1981 a 1989, quando as coletas de sangue foram realizadas entre $19 \mathrm{~h}$ e $21 \mathrm{~h}$, a prevalência média foi de 1,5\%. Em 1992, quando o diagnóstico foi baseado em coletas da gota espessa realizadas entre $22 \mathrm{~h}$ e $24 \mathrm{~h}$, a prevalência subiu para $6 \%$ (Tabela 2). Apenas com a mudança do horário de coleta, verificou-se um aumento considerável no número de microfilarêmicos, comprovando que os índices obtidos nas campanhas anteriores subestimavam a real prevalência da filariose nas áreas endêmicas.

Verificação semelhante ocorre quando comparamos os resultados de campanhas realizadas pela FNS, que mostravam um percentual de apenas 1,4\% de microfilarêmicos no Recife, nos anos de 1987 e 1988, (MS, 1983), com os resultados obtidos no período de 1989 e 1990. Nessa oportunidade, a campanha da FNS integrou-se à do Centro de Pesquisas Aggeu Magalhães/Fiocruz. O inquérito epidemiológico abrangendo quinze mil pessoas, realizado pelo CPqAM, cujas coletas eram realizadas entre $20 \mathrm{~h}$ e $24 \mathrm{~h}$ e com três gotas de sangue de uma mesma pessoa (60 4 l) em vez de apenas uma gota $(45 \mu \mathrm{l})$, resultou numa prevalência de microfilaremia média de 6,5\% para a cidade do Recife, atingindo $15 \%$ em algumas áreas da cidade (Maciel et al., 1996).

Dentre as conclusões apresentadas no workshop nacional sobre filariose bancrofti realizado em 1989 no Recife, foi sugerido que, a partir daquela data, as coletas de sangue realizadas para diagnóstico da filariose se inicias- 
sem no mínimo às $20 \mathrm{~h}$ e que se coletassem de cada indivíduo no mínimo três gotas de sangue (MS, 1989). No entanto, a FNS continua efetuando as coletas nos mesmos padrões tradicionais, ou seja entre $19 \mathrm{~h}$ e $21 \mathrm{~h}$ e apenas uma gota de sangue por indivíduo.

Há quase cinqüenta anos, a filariose é endêmica no Recife e persiste como um problema de saúde pública. Já atinge as populações dos Municípios de Recife, Olinda, Paulista e Jaboatão (Tabela 1). Jamais foi avaliado o impacto dos custos sociais e econômicos representados pelos portadores de formas agudas e crônicas da doença e pelos microfilarêmicos aparentemente subclínicos mas que podem ter alterações renais e/ ou linfáticas (hematúria, quilúria), detectáveis por exames laboratoriais. Necessitam igualmente de avaliação os custos do programa de controle para o estado e para o município, em termos de recursos humanos, operacionais, terapêutica e diagnóstico para uma completa eficácia e eficiência. O programa de controle deve ser avaliado sistematicamente, considerando novas abordagens clínico-laboratoriais, estratégias e ações factíveis para o município, já que, no que se refere ao nível estadual, a filariose não se inclui como prioridade de governo.

Como sugestões de estratégias de controle, teríamos:

1) Uso de sal medicado com DEC, durante o período de um ano, suficiente para o controle (WHO, 1988). Sua distribuição poderia ser controlada pelas associações de bairro, distribuídas em toda a região metropolitana do Recife. O custo estimado por adulto/ano é de US\$0,80 e para toda a população carente do Recife seriam necessários US\$72,800.00.

2) Tratamento em massa com esquemas variados de distribui ção da DEC. Poderia ser realizado utilizando-se os recursos humanos já existentes do Programa de Agentes Comunitários (Pacs).

3) Controle do vetor por meio de métodos alternativos, tais como camadas de pérolas de isopor em fossas, tratamento de criadouros com larvicidas à base das bactérias entomopatogênicas B. sphaericus e B. thuringiensi s var. israel ensis e peixamento de grandes criadouros com peixes larvívoros.

4) Controle integrado, associand o o tratamento em massa ao controle do vetor.

5) Capacitação dos técnicos de saúde para o diagnóstico da parasitose de qualquer indivíduo da área endêmica que apresente história clínica de febre recorrente, associada à adenolinfangite ou à manifestação isolada ou associada de linfedema, elefantíase, hidrocele e/ ou
Tabela 2

Prevalência de microfilaremia no período de 1981-1992, Recife, PE.

\begin{tabular}{lrrr}
\hline Ano & Lâminas coletadas & Lâminas positivas & \% dos positivos \\
\hline 1981 & 290.135 & 4.186 & 1,7 \\
1982 & 290.914 & 4.549 & 1,6 \\
1983 & 210.711 & 2.029 & 1,0 \\
1984 & 205.298 & 1.365 & 0,7 \\
1985 & 200.764 & 1.268 & 0,6 \\
1986 & 103.969 & 1.403 & 1,3 \\
1987 & 76.900 & 661 & 0,9 \\
1988 & 101.992 & 1.423 & 1,4 \\
1989 & 95.467 & 2.035 & 2,1 \\
$1990 *$ & 10.881 & 676 & 6,2 \\
1991 & 54.376 & 1.880 & 3,5 \\
$1992 * *$ & 27.266 & 1.638 & 6,0 \\
\hline
\end{tabular}

Fonte: MS, 1983, 1990, 1992.

* três gotas espessas de cada indivíduo, coletadas no horário das 21-24h.

** uma gota espessa, coletada de cada indivíduo após às $22 \mathrm{~h}$.

orquite. A partir disso, seria desencadeada a rotina de notificação, confirmação laboratorial, tratamento, seguimento e orientação dos familiares.

6) Trabal ho de controle permanente, integrado às comunidades e respectivos centros de saúde, através do Pacs, coordenado por equipe multidisciplinar e lideranças comunitárias.

7) Controle das formas agudas na comunidade, mais freqüentes na faixa etária de 15-25 anos; nas escolas municipais e estaduais de áreas carentes, tratamento semestral com DEC dos adolescentes de 12-15 anos.

O controle da endemia seria, portanto, papel do município. O medicamento específico, DEC, seria produzido no nível estadual e distribuído para a rede primária de saúde, desmistificando e descentralizando a endemia, objetivando contribuir a médio prazo para o seu controle.

Por meio da atenção primária, será possível realizar e executar estratégias de transformação do atual modelo assistencial de controle, não só da filariose, mas também de outras endemias que se superpõem em áreas urbanas, em razão dos critérios de eficiência e eficácia de novos programas, realizando um conjunto de ações intersetoriais orientadas para a implantação e evolução de ações descentralizadoras. Deve ser estimulada a busca de novas abordagens menos assistencial istas do que as campanhas do passado; as novas devem ser moldadas em propostas de base ecológica e visão interdisciplinar com fundamentação técnica e política para a construção de uma partici- 
pação comunitária efetiva (Marzochi, 1994). Não é possível esquecer que a mobilização de recursos humanos e técnicos, com envolvimento de grandes contigentes de população, acarreta custos que requerem avaliação. A filariose linfática disputa recursos financeiros com outras endemias e epidemias mais proeminentes. Os recursos nela investidos certamente contribuiriam para a redução e o controle de

\section{Referências}

AZEVEDO, R., 1955. Contribuição ao Estudo da Bancroftose. Tese apresentada à Faculdade de Medicina da Universidade do Recife para concorrer à vaga de professor catedrático de Doenças Tropicais e Infectuosas, Recife: Faculdade de Medicina da Universidade do Recife.

AZEVEDO, R. \& DOBBIN Jr., J. E., 1952. Filariose (Wuchereria bancrofti) no grupo residencial do IAPB no bairro de Afogados (Recife). Publicações Avulsas do Instituto Aggeu Magalhães, 1:157-192.

BARJAC, H. J. \& SUTHERLAND, D. J., 1990. Bacterial Control of Mosquitoes and Black Flies. London/ New Jersey: Rutgers University Press.

CABES (Catálogo Brasileiro de Engenharia Sanitária e Ambiental), 1994. Guia do Saneamento Ambiental no Brasil. Rio de Janeiro: Cabes.

COUTINHO, A. D.; DREYER, G.; MEDEIROS, Z.; LOPES, E.; MACHADO, G.; GALDINO, E.; RIZZO, J. A.; ANDRADE, L. D.; ROCHA, A.; MOURA, I.; GODOY, J. \& OTTESEN, E. A., 1994. Ivermectin treatment of Bancroftian Filariasis in Recife, Brazil. American Journal of Tropical Medicineand Hygiene, 50:339-348.

CURTIS, C. F., 1994. Approaches to vector control: new and trusted. Transactions of the Royal Society of Tropical Medicineand Hygiene, 88:144-146.

FRANCO, O. \& LIMA, D. H., 1967. Alguns aspectos das atividades contra a filariose bancroftiana no Brasil. Revista Brasileira de Malariologia e Doenças Tropicais, 19:73-89.

FURTADO, A. F.; MACIEL, M. A.; ROCHA, A; REGIS, L. N.; SILVA-FILHA, M. H.; BRAGA, C.; FERREIRA, R.; SILVA, M. C. M.; ALBU QUERQUE, M. F. M.; SOUZA, W. \& LAPA, T., 1994. Programa de controle da filariose na cidade do Recife. Relatório Anual da Fundação Oswal do Cruz, ano de 1993. Recife: Fundação Oswaldo Cruz.

GROVE, D. I., 1983. Selective primary health care: strategies for the control of diseases in the developing world. VII Filariais. Reviews of Infectious Diseases, 5:933-944. outras doenças endêmicas de consideráveis padrões de morbidade e letalidade.

Esta proposta mais ampla está voltada para o planejamento conjunto de controle das endemias mais preval entes numa mesma região ou área, onde varia o envolvimento de diferentes grupos profissionais, e interessaria mais às lideranças políticas nos seus diferentes níveis ao ampliar os resultados e reduzir os custos.
JAYASEKERA, N.; KALPAGE, K. S. P. \& SI LVA, C. S. S., 1991. The significance of the low density microfilaraemia in the transmission of Wuchereria bancrofti by Culex (Culex) quinquefasciatus Say in Sri Lanka. Transactions of the Royal Society of Tropical Medicine and Hygiene, 85:250-254.

MACIEL, M. A. V.; SILVA, E. C.; ROCHA, A.; MARZOCHI, K. B. \& FURTADO, A. F., 1994. Estudo comparativo de filariose bancroftiana em região metropolitana do Recife. Cadernos de Saúde Pública, 10(Sup. 2):301-309.

MACIEL, A.; ROCHA, A.; CARVALHO, A. B.; SOUZA, W. \& FURTADO, A. F., 1996. Epidemiology of Wuchereria bancrofti in Recife, Northeast of Brazil. Memórias do Instituto Oswaldo Cruz, 91: 449-455.

MARZOCHI, K. B. F., 1994. Dengue in Brazil - situation, transmission and control - a proposal for ecological control. Memórias do Instituto Oswaldo Cruz, 89:235-245.

MAXWELL, M. S.; CURTIS, C. F.; HAJI, H.; KISUMKU, S.; THALIB, A. I. \&YAHYA, S. A., 1990. Control of Bancroftian filariasis by integrating therapy with vector control using polystyrene beads in wet pit latrines. Transactions of the Royal Society of Tropical Medicine and Hygiene, 84:709-714.

MEDEIROS, Z.; DREYER, G. D.; ANDRADE, L.; PIRES, M. L.; MENDES, J. \& PIMENTEL, R., 1992. Wuchereria bancrofti, microfilarial density of auchtothonous cases and natural Culex infectivity rates in Northeast Brazil. American Journal of Tropical Medicineand Hygiene, 95:214-217.

MORAIS, H. M., 1982. A Filariose no Recife: Um Estudo Epidemiológico. Dissertação de Mestrado, São Paulo: Faculdade de Medicina, Universidade de São Paulo.

MS (Ministério da Saúde), 1983. Controle das Endemias em 1983. Brasília: Superintendência de Campanhas de Saúde Pública.

MS (Ministério da Saúde), 1989. Workshop Nacional sobre Filariose Bancrofti. Documento Final. Re- 
cife: Superintendência de Campanhas de Saúde Pública, Diretoria Regional da Saúde.

MS (Ministério da Saúde), 1990. Programa de Controle da Filariose. Rel atório Anual. Recife: Superintendência de Campanhas de Saúde Pública, Diretoria Regional da Saúde.

MS (Ministério da Saúde), 1992. Programa de Controle da Filariose. Boletim Anual. Brasília: Fundação Nacional de Saúde.

MS (Ministério da Saúde), 1994. Descentralização do Controle de Endemias. Brasília: Fundação Nacional de Saúde.

MULLA, M. S.; DARWAZEH, H. A.; DAVIDSON, E. W.; DULM AGE, H. T. \& SINGER, S., 1984. Larvicidal activity and field efficacy of Baccilus sphaericus strains against mosquito larvae and their safety to nontarget organisms. Mosquito News, 44:336342.

OTTESEN, E. A., 1985. Efficacy of diethylcarabamazine in erradicating infection with lymphaticdwelling Filariae in humans. Reviews of Infectious Diseases, 7:341-351.

OTTESEN, E. A. \& RAM ACHADRAN, C. P., 1995. Lymphatic filariasis infections and disease: control strategies. Parasitology Today, 11:29-31.

RACHOU, R. G., 1954. Índices epidemiológicos em inquéritos de filariose bancroftiana. Revista Brasileira de Malariologia e Doenças Tropicais, 6:3540.
RACHOU, R. G., 1957. Distribuição geográfica das filarioses humanas no Brasil. Revista Brasileira de Malariologia e Doenças Tropicais, 9:79-100.

RACHOU, R. G. \& SCAFF, L., 1958. Redução da microfilaremia de Wuchereria bancrofti com Hetrazan em esquema de tratamento de duração variável. Revista Brasileira de Malariologia e Doenças Tropicais, 10:303-325.

REGIS, L. N.; FURTADO, A. F.; SILVA-FILHA, M. H. N. L.; OLIVEIRA, C. M. F. \& SI LVA, S. B., 1994. The impact of Bacillus sphaericus treatment in Culex quinquefasciatus population in urban areas of Recife, Brazil. Proceedings of theVI International Colloquium in Invertebrate Pathology and Biological Control, 1:254-258. Montpellier.

REGIS, L.; SILVA-FILHA, M. H. N. L; OLIVEIRA, C. M. F.; RIOS, E. M.; SILVA, S. B. \& FURTADO, A. F., 1995. Integrated control measures against Culex quinquefasciatus, the vector of filariasis in Recife. Memórias do Instituto Oswal do Cruz, 90:115-119.

SES (Secretaria Estadual de Saúde), 1996. Proposta de Intervenção sobre a Filariose Linfática em Pernambuco. Recife: Secretaria do Estado de Pernambuco.

SCAFF, L. M. \& GUEIROS, Z. M., 1967. Prevalência e controle da filariose no Pará: estado atual. Revista Brasileira de Malariologia e Doenças Tropicais, 19:245-52.

WHO (World Health Organization), 1988. Control of Lymphatic Filariasis (A Manual for Health Personnel). Geneva: WHO. 